\title{
Measurement of the cosmic ray rate as a function of the altitude and atmospheric variables using a portable detector
}

\author{
M. I. Martínez, E. Moreno Barbosa*, R. Palomino, M. Rodríguez Cahuantzi and G. \\ Tejeda, \\ Facultad de Ciencias Físico Matemáticas, Benemérita Universidad Autónoma de Puebla, \\ Ciudad Universitaria, Colonia San Manuel Puebla, México \\ E-mail: mario.rodriguez@correo.buap.mx
}

\begin{abstract}
The secondary flow of particles produced in the atmosphere by galactic and extragalactic primary cosmic rays is affected by the Earth's magnetic field and by atmospheric variables such as pressure and temperature. In this work measurements of the cosmic ray rate obtained with three counting detectors are shown. The detectors consist of three plastic scintillators coupled to photomultiplier tubes. The output pulses from the photomultiplier tubes were discriminated and counted by a custom data acquisition board. Measurement of the altitude and atmospheric pressure was made with a minimum system (raspberry Pi). Correlations between the measured rate of particles with altitude and pressure are presented for two scintillator plastics made at the laboratory and one commercial sample. Measurements were made at several altitudes from 0 m.a.s.1., in the state of Veracruz, Mexico up to 4530 m.a.s.l. at the top of the Sierra Negra mountain.
\end{abstract}

35th International Cosmic Ray Conference - ICRC2017

10-20 July, 2017

Bexco, Busan, Korea

\footnotetext{
${ }^{*}$ Speaker.
} 


\section{Introduction}

The Earth is being continuously bombarded by cosmic radiation (CR) from external sources. $\mathrm{CR}$ is composed of subatomic particles coming from outer space with energies from $10^{13}$ up to $10^{21} \mathrm{eV}$ per particle. It is known that the composition of primary cosmic rays is aproximately $90 \%$ protons, $9 \%$ He nuclei and $1 \%$ of heavier components. At low energies the composition of the primary cosmic rays is dominated by protons with a few percentage of He nuclei while at higher energies the nuclear contribution increases [1]. The existence of such kind of radiation has been known for almost a century but the origin and mechanisms of production of these particles with energies larger than $10^{18} \mathrm{eV}$ have not yet been determined and have motivated the development of increasingly sophisticated particle detectors $[2,3]$.

To measure the CR rate with respect to the altitude, we have arranged a setup consisting of 3 particle detectors based on plastic scintillator coupled to fine mesh Hamamatusu PMT sensors operated to an optimal HV value. Two scintillation detectors were fabricated at the materials science laboratories of the University of Puebla and the University of Sinaloa. The third detector was built using a commercially available plastic scintillator from Bicron. In this work we measured the CR rate at altitudes ranging from sea level up to 4530 m.a.s.l. We also compared the performance of the two "lab-made" scintillators with respect to the commercial sample.

\section{Materials and Methods}

The three pieces of scintillation material used in this work are shown in Figure 1. The piece made at University of Puebla (referred to as BUAP from now on) has a radius of $9.5 \mathrm{~cm}$ and is 2.54 $\mathrm{cm}$ thick. Concentrations of $1.5 \%$ of POPOP and $0.02 \%$ of PPO were used in the fabrication of this sample. The dimensions of the piece made at the University of Sinaloa (referred to as UAS from now on) are $14 \times 15 \times 2 \mathrm{~cm}^{3}$. To produce this counter concentrations of $0.01 \%$ for POPOP and $1 \%$ of PPO were used. The commercial sample is a $18 \times 22 \times 2.5 \mathrm{~cm}^{3}$ piece of Bicron's BC 404 . The cutout at the corner ${ }^{1}$ has a radius of $6 \mathrm{~cm}$. The plastic pieces were coupled to the same type of photomultiplier tube (Hamamatsu R5946, hybrid assembly H6153-70) in order to compare the performance of the scintillator materials.

The plateau curve was obtained for each detector (see Figure 2) to determine an optimum operating voltage for each one $[4,6]$.

A 4-channel discrimination and counting board (developed at the electronics laboratory at University of Puebla)[5] was used to register the counting rate of the three detectors. Measurements were taken every 500 meters starting at the top of the Sierra Negra mountain (at 4530 m.a.s.l) down to sea level at the city of Boca del Rio, Veracruz. Measurements of altitude and atmospheric pressure were performed by means of the BMP085 sensor and a minimum processing system (raspberry PI V3.0). These atmospheric variables were correlated to the measured counting rate.

\section{Results}

Figure 3 shows the variation of pressure and altitude during the course of the day as we mea-

\footnotetext{
${ }^{1}$ This piece was recovered from another experiment so its particular shape.
} 


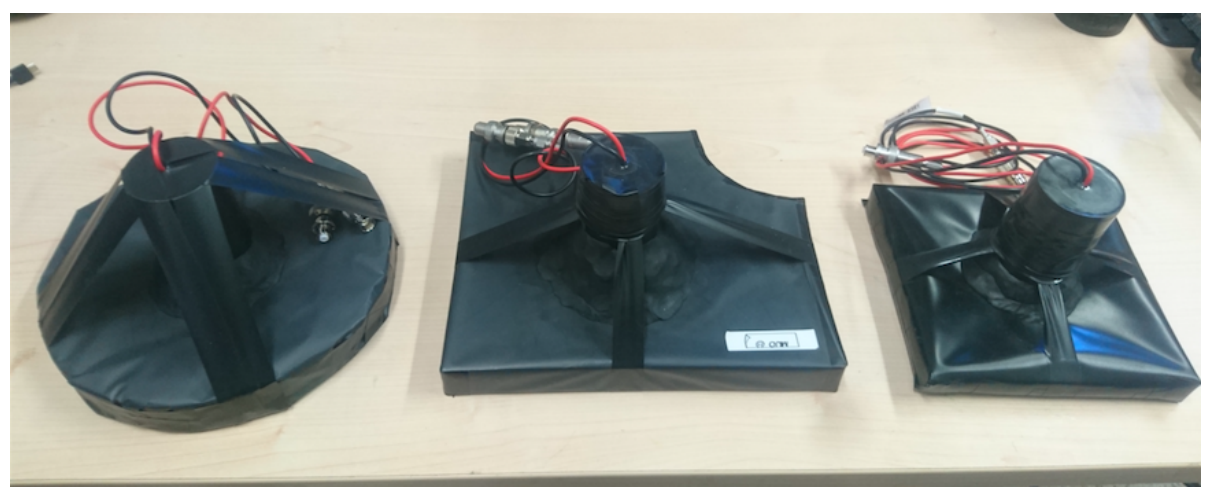

Figure 1: Scintillator detectors used in this work: BUAP (left), Bicron BC404 (center) and UAS (right). Optical grease was used to improve the coupling between the PMT's (seen at the center of each detector) and the plastic pieces.

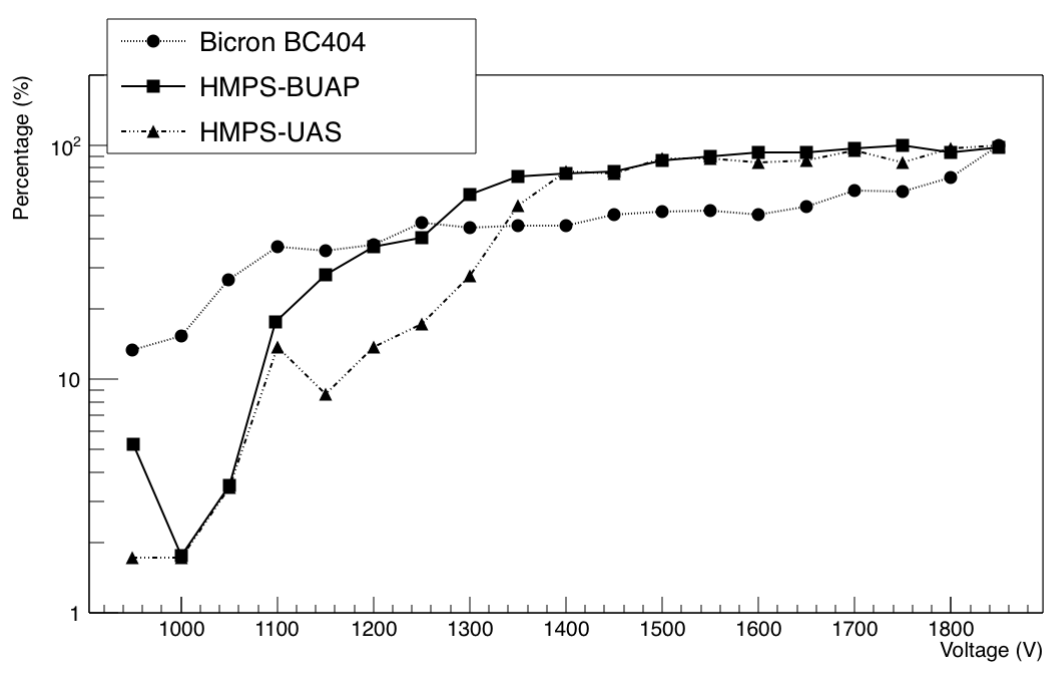

Figure 2: Plateau curve of the three scintillator systems. We used $1550 \mathrm{~V}$ for BUAP, $1600 \mathrm{~V}$ for UAS and $1500 \mathrm{~V}$ for Bicron.

sured the CR rate from the top of the mountain down to sea level. The points before the highest altitude correspond to the trip from the city of Puebla (at around $2000 \mathrm{~m}$ ). The expected correlation between altitude and pressure can be seen in these plots and in the plot at the bottom of Figure 4.

The CR rate measured by the three detectors as a function of altitude is shown in Figure 4, top. We used the commercial counter as a reference to evaluate the performance of the BUAP and UAS scintillators. For this purpose the actual rates measured with the BUAP and UAS counters were weighted using the ratio of the volume of the BC404 to their respective volume. This accounts for the different area and thickness of the three detectors. The rates for BUAP and UAS shown in Figure 4 have this factor applied.

The BUAP and UAS counters show similar behavior except for the measurement at $4530 \mathrm{~m}$ where the UAS detector gave a significantly lower rate than the BUAP detector. On the other hand 

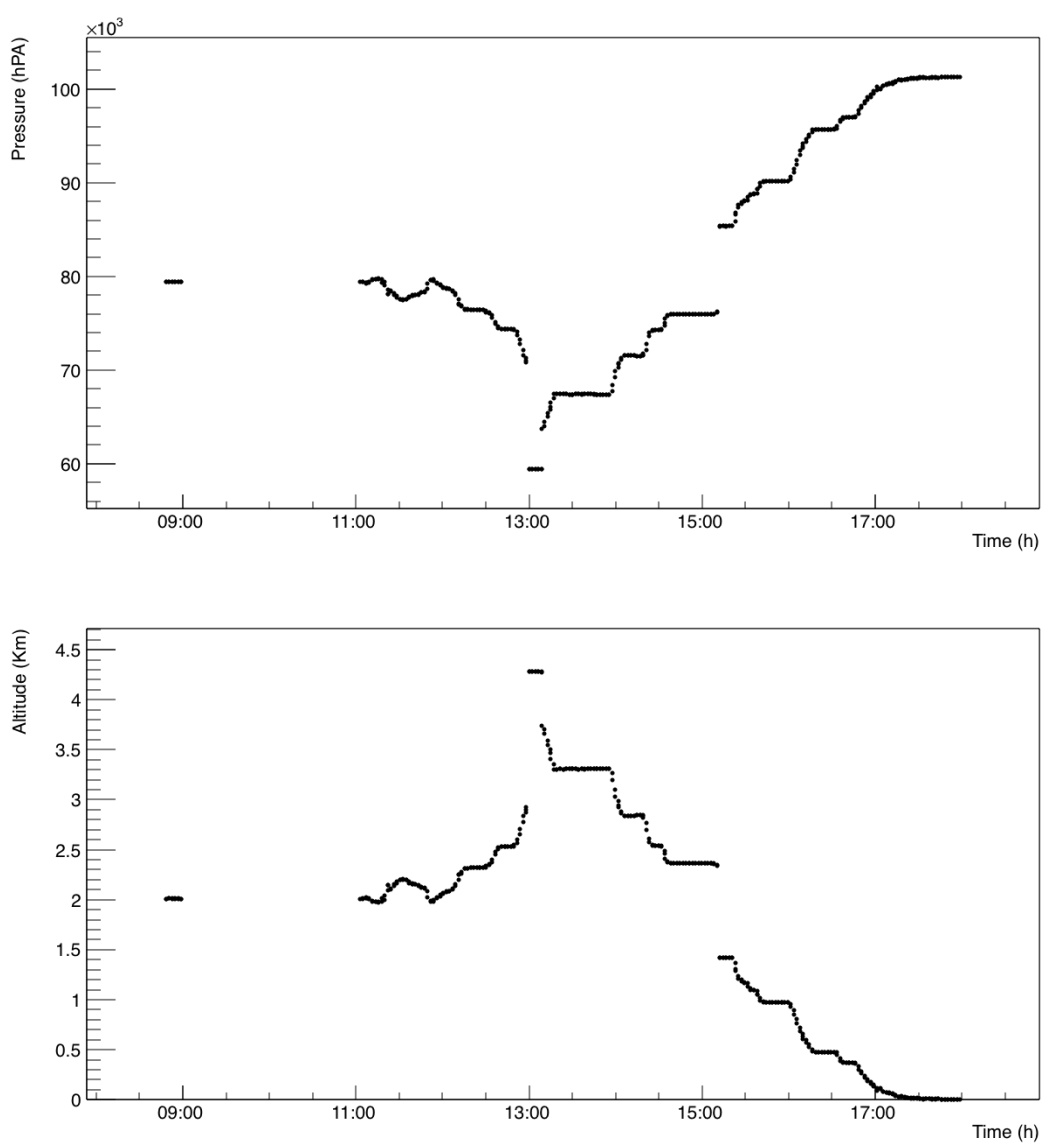

Figure 3: Atmospheric pressure (top) and altitude (bottom) measured with the raspberry Pi sensor. The expected variation of pressure with altitude can be seen clearly. Data taken before the first rate measurement (at the highest point) are also shown.

when comparing these detectors with the $\mathrm{BC} 404$ we observe their rates are lower by a factor of about 2 at low altitudes. However at the highest point the rate measured with the BUAP counter is comparable to the one obtained with the BC40w4.

\section{Conclusions}

As the top plot in Figure 4 shows an exponential rise in the CR rate with increasing altitude can be observed for the three systems tested. The rate measured with the UAS counter at the highest point is lower than expected. This detector showed a similar reduced rate in previous tests carried out during the characterisation phase using an LED at a relatively high pulse rate. We believe this could be explained by a long recovering time of the scintillating material used to fabricate the UAS counter given the high rate involved in both cases. Clearly a more detailed study should be made using, for example, increasing light pulse rates from the LED to reproduce the drop of the counting rate of this detector. 

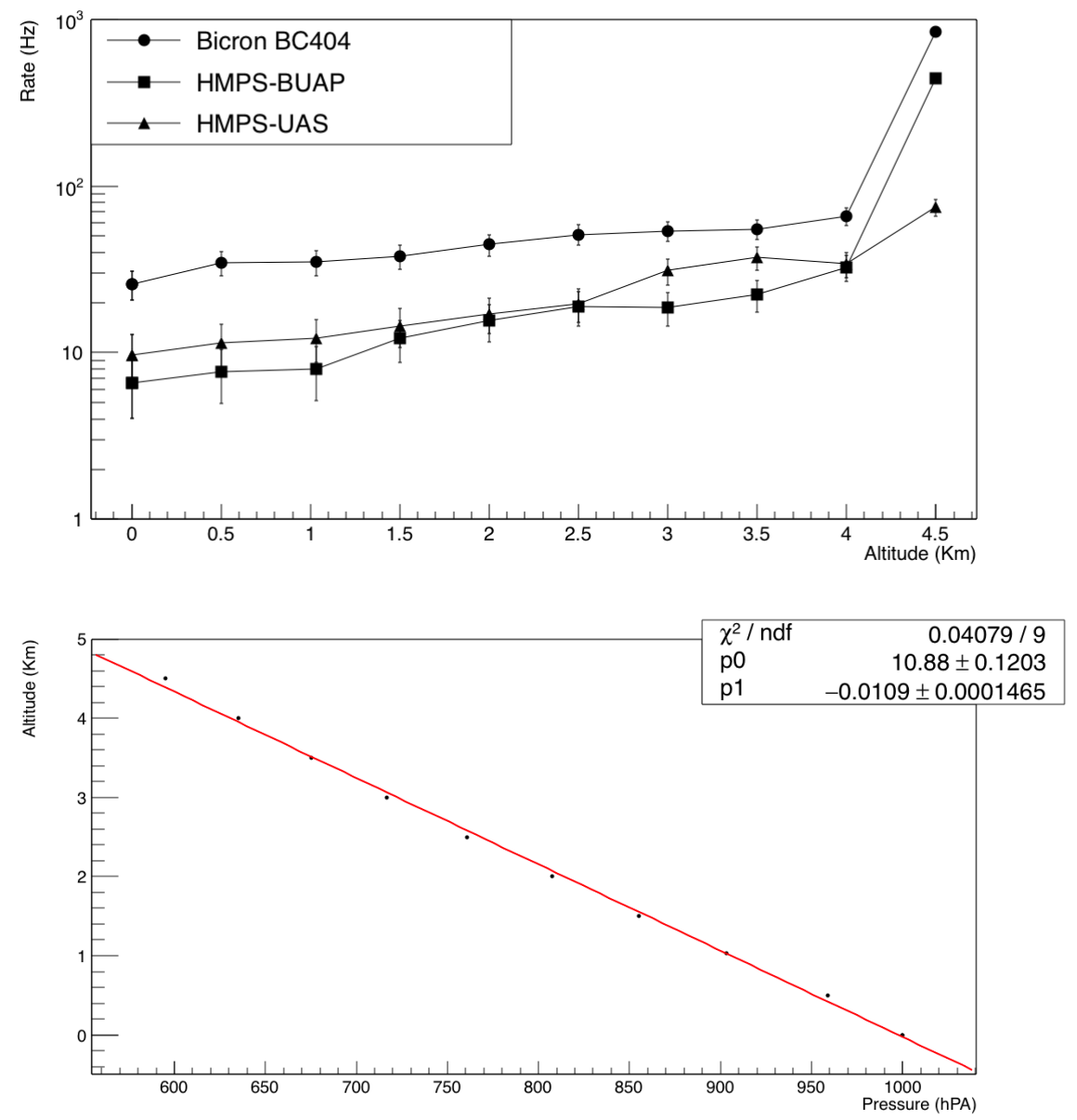

Figure 4: Rate measured with the three detectors (top) and atmospheric pressure (bottom) for the ten altitude points.

The average ratio of the rate measured with the BUAP detector to the rate measured with the BC404 is 50\% for all altitudes. On the other hand for the UAS detector we also get a 50\% average ratio for altitudes up to $4000 \mathrm{~m}$. However this ratio dropped to $13 \%$ at the highest altitude.

The change in the slope of the rate versus altitude curves at an altitude of $4000 \mathrm{~m}$ (Figure 4, top) suggests measuring at closer points between 4000 and $4530 \mathrm{~m}$. This should describe more accurately the exponential rise.

\section{Acknowledgments}

The authors wish to thank the useful collaboration and support of the laboratories of Materials Science, Particle Detectors and Medical Physics at the Facultad de Ciencias Físico Matemáticas, BUAP. We also thank Dr. Ildefonso León from Universidad Autónoma de Sinaloa and Dr. Jorge Cotzomi from Benemérita Universidad Autónoma de Puebla for fruitful discussions that contributed to this work. 


\section{References}

[1] K. A. Olive et al. (Particle Data Group), The Review of Particle Physics, Chin. Phys. C 38090001 (2014) and 2015 update.

[2] N. V. Ampilogov et al. Large Area scintillation muon hodoscope for monitoring of atmospheric and heliospheric processes, Astrophys. Space Sci. Trans. 7 435-438

[3] F. Blanco, P. La Roca and F. Riggi. Cosmic rays with portable Geiger counter: from sea level to airplane cruise altitudes, Eur. J. Phys. 30 685-695

[4] S. Vergara et al. Characterization of the ACORDE scintillator counters using a PCI electronic card, Rev. Mex. Fis. 53 120-125.

[5] 2012, patent $M X / a / 2012 / 001505$

[6] HAMAMATSU (2007), Photomultiplier tubes, Basics and Applications, https://www.hamamatsu.com/resources/pdf/etd/PMT_handbook_v3aE.pdf 